\title{
El principio de la didáctica de la ciencia. Una investigación narrativa
}

\author{
The principle of Science Didactics. A Narrative Investigation ${ }^{2}$ \\ O princípio da didática da ciência. Uma pesquisa narrativa ${ }^{3}$
}

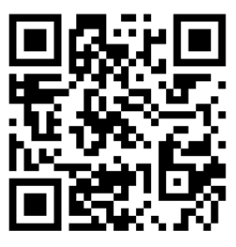

Víctor Amar-Rodríguez

Universidad de Cádiz

Cádiz, España

victor.amar@uca.es

https://orcid.org/0000-0001-9036-2651

Recibido $\cdot$ Received $\cdot$ Recebido: 19 / 11 / 2019
Corregido $\cdot$ Revised $\cdot$ Revisado: 10 / 07 / 2021
Aceptado $\cdot$ Accepted $\cdot$ Aprovado: 12 / 08 / 2021

\begin{abstract}
Resumen:
Objetivo. La didáctica de la ciencia ha experimentado una considerable evolución. Y, en este sentido, el objetivo es explicar de otra manera, si cabe, los principios de la ciencia para lo cual se vale de la didáctica, la filosofía de la ciencia y, también, la narrativa. Metodología. La opción para conocer y comprender lo que el personal docente universitario, vinculado a esta área de conocimiento, piensa, vive o siente en relación con la ciencia y su didáctica es la narrativa. Con todo, la información de corte cualitativa inspirada en el diálogo y la subjetividad explica la experiencia profesional de este profesorado. Análisis de resultados. De este modo, se hace imprescindible saber interpretar el conocimiento científico para comprender el mundo y que los sujetos no se conviertan en neoexcluidos del siglo XXI. Se presenta una propuesta de didáctica de la ciencia de manera integral y que no establezca separación entre ciencia y humanidades, ya que la propia ciencia es una construcción humana y no un producto. $Y$, es por ello, que se plantea la necesidad de alfabetizar y educar, científicamente, a la ciudadanía de forma proactiva y participativa en conexión con la vida actual. Conclusión. Se presenta la necesidad de desarrollar un modelo de cultura científica para crecer como persona y que sea capaz de generar un conocimiento cercano a los valores (sociales y ambientales), alejado de la educación instrumental o de la instrucción. Se aboga por una educación y saber científico que supere el modelo transmisible por otro más comprensivo y cotidiano.
\end{abstract}

Palabras claves: Didáctica de la ciencia; educación científica; narrativa; saber científico; alfabetización científica.

\footnotetext{
${ }^{1}$ Este artículo deriva del proyecto de investigación Proyecto de Innovación y Mejora Docente dentro del marco de actuaciones de las actuaciones avaladas. Universidad de Cádiz.

2 This paper derives from the research project "Innovación y Mejora Docente" (Innovation and Teaching Improvement) in the framework of endorsed interventions. University of Cadiz

${ }^{3}$ Este artigo deriva do projeto de pesquisa Proyecto de Innovación y Mejora Docente dentro del marco de actuaciones de las actuaciones avaladas. Universidad de Cádiz.
} 
http://doi.org/10.15359/ree.25-3.29

http://www.una.ac.cr/educare

educare@una.ac.cr

\begin{abstract}
:
Objective. Teaching science has undergone considerable development, and, in this sense, the objective is to explain, otherwise if possible, the principles of the science, making use of the didactics, philosophy of science or, also, the narrative. Methodology. The methodology chosen was to know and understand what university teachers linked to this area of knowledge think, live, or feel about science and didactics. In this sense, the qualitative information inspired by the dialogue, the personal or social-educational life lessons explains and gives to understand, differently, counting on the own experience and subjectivity of the informant. Analysis of results. However, in relation to the analysis of results, it is essential to know how to interpret scientific knowledge in order to understand the world and not to become neo-excluded individuals from the 21st century. We present a didactic proposal of science in an integral way; that not to set abysmal gaps between science and humanities, because science itself is a human construction and not a product. And, for this reason, we raised the need for literacy and educate, scientifically, to the proactive and participatory citizenship in connection with life. Conclusion. We present the need to develop a model of scientific culture to grow as a person and generate knowledge close to the values (social and environmental), and away from instrumental or instruction. So, we stand for education and scientific knowledge that exceeds the transmissible model by another more comprehensive and quotidian one.
\end{abstract}

Keywords: Science didactics; scientific education; narrative; scientific knowledge; scientific literacy.

\title{
Resumo:
}

Objetivo. A didática da ciência tem experimentado um considerável desenvolvimento. $E$, neste sentido, o objetivo é explicar de outra maneira, se é possível, os princípios da ciência através da didática, a filosofia da ciência e, também, a narrativa. Metodologia. A metodologia narrativa será nossa opção para conhecer e compreender o que um docente universitário, vinculado a esta área de conhecimento, pensa, vive ou sente em relação com a ciência y sua didática. Embora, a informação de corte qualitativa inspirada no diálogo e a subjetividade, explica a experiência profissional deste professor. Análise de resultados. Assim, é imprescindível saber interpretar o conhecimento científico para compreender o mundo e que os sujeitos não sejam os neo excluídos do século XXI. Apresenta-se uma proposta de didática de modo integral e que não chegue a estabelecer separação entre ciência e humanidades, já que a própria ciência é uma construção humana e não um produto. E, então, é preciso alfabetizar e educar, cientificamente, à cidadania de forma proativa e participativa em conexão com a vida atual. Conclusão. Se apresenta a necessidade de desenvolver um modelo de cultura científica para crescer como pessoa capaz de gerar um conhecimento próximo dos valores (sociais e ambientais) e longe da educação instrumental o da instrução. Quer dizer, se pensa em uma educação e saber científico que supera o modelo transmissivo por outro mais compreensivo e cotidiano.

Palavras-chave: Didática da ciência; educação científica; narrativa; saber científico; alfabetização científica. 


\section{Introducción}

En este contexto inicial haremos referencia al propio enunciado del artículo El principio de la ciencia. Este concepto de principio tiene varias acepciones. Por ejemplo, el de comienzo (a modo de punto de arranque o base), o bien el de una proposición fundamentada (por dónde empezar a estudiar o conocer un pensamiento). En este caso, utilizaremos ambos significados para nuestra propuesta y, para ello, planteamos iniciar un debate abierto, en continua construcción, con la intención de analizar, conocer y dar a comprender el parecer del personal docente sobre qué piensa, cómo vive o siente la ciencia (experimental) y su didáctica (Vázquez-Bernal et al., 2019). Una investigación inspirada en la metodología cualitativa, de corte narrativo, donde la palabra de la persona informante se empodera y da sentido a nuestra labor. Y su parecer nos interesa, tal como nos lo narra, pues su subjetividad y entender serán validadores de nuestra propuesta metodológica y de la investigación en sí.

Igualmente, en este intento por introducir la temática, digamos que un libro de cierto interés para aproximarnos a la ciencia puede que sea el coordinado por Michel Sandoval y García Guerrero (2014) titulado La ciencia en nuestras manos. Una perspectiva de los talleres de divulgación sin el color de rosa, que contó con el apoyo del Consejo Nacional de Ciencia y Tecnología de México. En este sentido, hay varios aspectos que nos llamaron la atención. El primero de ellos, la invitación a descubrirla, tocando; en segundo lugar, la metodología procesual del taller, también palpando y, por último, la intención divulgativa con el propósito de prolongarla sobre los demás, para su descubrimiento, extendiendo la mano. Ciertamente, notamos una intención por poner en movimiento a las ciencias empíricas. Ya no vale aquel principio de dificultad, pues ha quedado superado lo obtuso que sembraba el temor en el estudiantado, y ahora lo que priva es la seducción, una pedagogía persuasiva que lleve al alumnado a descubrirla para amarla, o viceversa.

La escuela o el instituto de enseñanza secundaria han cambiado la perspectiva de la didáctica de la ciencia, o están en su empeño. Tal vez, no queramos ver la evolución notable que han registrado. Pero en la propia sensibilidad por abordar a la ciencia han hecho de ella algo vivo, en nuestras manos y para su comprensión. Con ello, la acción de comprender lo interpretamos como el proceso de un conjunto de cualidades que facultan la capacidad de entender el hecho científico alcanzando la justificación de lo qué y cómo, además de para qué, por qué, cuándo o para quién. Dar a comprender pasa por la premisa de una ciencia para la indagación (enseñanza de las ciencias basada en la indagación -ECBI-) (Aguilera Morales et al., 2018), donde el sujeto aprendiz ha de ser activo y no un mero receptor de unos contenidos obsoletos o abstractos, de difícil entendimiento y descontextualizado. La indagación mejora la motivación y el interés y, por consiguiente, el aprendizaje. Estamos ante una propuesta de aprendizaje práctico, donde el alumnado pueda participar de la construcción de modelos o proyectos de investigación, argumentando y escribiendo sobre la ciencia, en el laboratorio, en el aula o en la casa, según necesidades de materiales, apoyo o adaptación (Galagovsky Kurman, 2011). 
http://doi.org/10.15359/ree.25-3.29

http://www.una.ac.cr/educare

educare@una.ac.cr

Sobre este parecer, consideramos que el hecho investigativo se centra en dar respuesta a estas cuestiones discurriendo o a través de preguntas-respuestas que, tras un proceso de planificación de la investigación, buenos interrogantes, búsqueda de información, o bien por la vía del análisis-reflexión se llegue a la elaboración de argumentos coherentes evaluados (con la intención de conocer) y valorados (con la finalidad de establecer posibles respuestas). La idea que estamos empezando a compartir es que el personal docente tiene su responsabilidad en que el alumnado participe del saber, la práctica y, también, de los fenómenos científicos a partir de la indagación (Simon Auerbach, 2019). Esta corresponsabilidad se desarrolla a través de la indagación con un propósito: a) estructurado, donde se presentan las preguntas y procedimientos a seguir y es el alumnado quien explica los resultados, b) guiado, con una presencia del profesorado que facilita la pregunta y el estudiantado es quien genera el procedimiento y c) abierto, ante un alumnado emancipado que genera la pregunta según intereses, estructura el procedimiento, investiga y es capaz de comunicar los resultados.

Estamos ante maneras de comprender y dar a comprender la ciencia y sus posibles explicaciones, un ejercicio idóneo para analizar y reflexionar sobre el conocimiento científico. Es decir, hablamos del principio de cultura científica para dar respuesta a las demandas actuales, que vinculamos al aprendizaje cooperativo (Ovejero Bernal, 2018), un buen pretexto para participar activa y productivamente del saber científico. En este, el hecho comunicativo se hace imprescindible para que la actividad tenga sentido. Y, es por ello, que el conocimiento y tratamiento del lenguaje son necesarios para poder conectarlo con las coordenadas culturales de pertenencia, en pro de la propia comunidad en la que se aprende, procurando la interacción social (Ballesteros, Velázquez, 2019).

La ocasión de conocer es una invitación para investigar sobre estos contenidos científicos que ya atesoran una larga tradición nacional como internacional (Acevedo Díaz, 2010; Arteaga Valdés et al., 2016; de Pro Chereguini et al., 2020; Parker, 2006). Estamos ante un modelo de coconstrucción de la ciencia dinámica y participativa; contribuyendo un saber tecnocientífico fiel a las consignas del aprendizaje significativo, ampliando y aplicando situaciones nuevas para la didáctica de la ciencia. La idea de que el alumnado sea el hacedor del proceso se significa desde el momento en que se le permite relacionar conceptos, informaciones o contenidos con su saber apriorístico, condicionado por su experiencia. Y tras un proceso de maduración ha de modificar o reestructurar lo aprendido. Lo nuevo, ese dato o información, entra en interacción con aquel supuesto concepto relevante que interesa al estudiantado. En este aprendizaje previo se aúnan los conocimientos que va adquiriendo en el proceso, que han de fusionarse, creando una red de conexiones, generando un resultado nuevo a modo de saber.

Fiel al contexto del alumnado, otro aliciente en este modelo de aprendizaje es la capacidad que tenga el personal docente de presentar la ciencia, además de mostrar sus posibles relaciones y finalidades. Lejos queda el conocimiento memorístico, lo que Freire (2005) llamó, de saber 
http://doi.org/10.15359/ree.25-3.29

bancario. Igualmente, la repetición se diluye por la indagación y emana un nuevo resultado en el aprendizaje. Lo que prima es lo que tiene sentido para el sujeto aprendiz. El entorno de interés se ha de apoderar de la dinámica del aula y cada docente ha de tener capacidad para ilusionar en su labor de enseñante. De este modo, el aprendizaje se torna un disfrute, al igual que un hecho comprensivo. Por ello, otro acicate más podría ser, e insistimos en ello, el aprendizaje cooperativo; como una acción que se emplea en proponer un quehacer común. En este sentido, Pujolàs Maset (2008) afirma:

Debemos pensar que no todo el mundo tiene las cualidades específicas para saber trabajar en equipos reducidos, y trabajar en equipo es más difícil de lo que parece inicialmente. Incluso, con algunos alumnos (muy individualistas o competitivos) es muy difícil logarlo. De esta manera hemos constatado que se cae fácilmente en una especie de círculo vicioso: como que no saben trabajar en equipo y las veces que lo hemos probado no ha acabado de funcionar, casi no usamos esta forma de enseñar, o no la usamos nunca, con lo cual no aprenden jamás a trabajar en equipo. (pp. 215-216)

En un claro alarde iremos a reivindicar el humanismo en la enseñanza de la ciencia. Rompemos aquel vínculo exclusivo de herencia del pasado de presentar la ciencia aislada, descontextualizada y sin valor social. Nuestra consideración se centra en hacer valer la mayoría de las cualidades propias de la naturaleza humana de carácter crítico y ético, y que superan el adoctrinamiento y el fideísmo. El ser humano, individual o en comunidad, junto con la razón, adquiere relevancia. Y el pensamiento, en conjunción con la cultura y la creatividad, se activan (Blanco, 2015), lo que visa posibilidades de autorrealización y proyección social.

En este sentido, esta investigación está estructurada en cuatro apartados comunicados entre sí que se van complementando. O sea, introducción, metodología, resultados y conclusión.

\section{Metodología}

\section{Generalidades, objetivo y cuestiones de investigación}

Nuestra opción metodológica es de corte cualitativo-narrativa. Una elección por la que nos inclinamos, después de diferentes avatares y replanteamientos discursivos, que han hecho reconsiderar nuestro ámbito y sentido de la investigación en el campo de las ciencias sociales. Es, por ello, que hemos abrazado esta metodología compartiendo la voz y la lucidez de nuestro sujeto informante.

A todas luces, con este diseño metodológico lo que se trata es dar respuesta al objetivo de conocer y comprender lo propuesto por un profesor universitario sobre las ciencias experimentales y su didáctica. Es decir, conocer su opinión y sensibilidad, además de pretender llegar a comprenderlas (en referencia a la ciencia y a la didáctica) con la intención de compartirla 
http://doi.org/10.15359/ree.25-3.29

http://www.una.ac.cr/educare

educare@una.ac.cr

con las demás personas (haciéndose extensibles a ámbitos políticos y económicos, sociales y ambientales). Estamos ante una realidad transmitida, valorada y considerada, tanto por el informante, el investigador $y$, a partir de este momento, también por el público lector. Nos encontramos frente a una labor de investigación que reconoce la complejidad de los hechos narrados otorgándoles una visión abierta y en continua construcción.

Y, con ello, proponemos las siguientes cuestiones de investigación para dar respuesta al propio estudio (Decuir-Gunby y Schutz, 2017):

- ¿Qué papel desempeña la didáctica de las ciencias?

- ¿Se imparte correctamente los contenidos para una idónea educación científica?

- ¿Cuál es el grado de saber científico en el alumnado o la ciudadanía?

En definitiva, la investigación de corte narrativa no pretende establecer pleitesía ante el discurso dominante y homogéneo, donde se analiza las causas de las rupturas de las relaciones entre lo actual y lo ideal entre conocimiento y poder (Harding, 2007). El propósito es reivindicar no solo un estadio de información sino, también, de conformación y confrontación de la realidad a través de la comprensión. No estamos ante un conocimiento investigado de carácter absoluto o exportable. Este es cuestionable y compartido y, por tanto, variable y mejorable.

\section{Subjetividad y discurso}

A pesar de todo, el diseño de investigación obedece a unos postulados dialógicos, siendo el objeto de estudio la propia subjetividad de la persona entrevistada. Es decir, una subjetividad entendida como una expresión vinculada a la propia persona en relación con la manera de pensar, sentir y actuar (Arfuch, 2007). Sin soslayar que en ella hay influencia de los intereses de quien narra (inclusive omisiones u olvidos de la memoria) y sus deseos particulares (hacer hincapié o explicar detenidamente algo que le preocupa o ambiciona).

Estamos ante un modelo de investigación inspirado en el dar a comprender unas realidades subjetivas (Moriña, 2017). Con ello, el diálogo, a partir de conversaciones compartidas, será parte imprescindible para la investigación. Pues reivindicamos el conocimiento situado, además de la validez del sujeto epistemológico y no subordinado. Y, en este caso, compartimos la voz con un profesor universitario, supuestamente, una razón académica que piensa, habla y siente. Pero, ahora, él será el motivo de estudio. Un intento por presentar una versión personal de unos hechos narrados, junto con una sensibilidad generosa, que hacen que cuestionemos el conocimiento y la didáctica de la ciencia. Una visión contextualizada, donde se llegan a debatir tópicos con una mirada crítica. Una manera de conectar la metodología con las intenciones iniciales de presentar una ciencia más humanista; pues la voz está en manos del sujeto informante, quien adquiere la calidad y el reconocimiento de sujeto activo que piensa y hace, dice y siente en un contexto personal y social, educativo y didáctico.

6 
Estamos ante un modelo de investigación donde lo expuesto puede (o ha de) ser transformado a partir de la valoración de la subjetividad. Lo que interesa no es, en exclusiva, centrifugar la verdad de lo narrado, sino que, igualmente, lo apreciable es hacer acopio de cómo se transmite y sus significados en este contexto discursivo. Con un resultado final en forma de texto con incrustaciones de información adicional de otros estudios e interpretaciones agregadas de quien suscribe el texto, con el propósito de nutrir el relato. No son simples aportaciones. En este sentido, cabría interpretarse como acciones discursivas en torno a la realidad que pertenecen al sujeto que permiten un acercamiento y conocimiento de su verdad, que construyen y constatan la realidad. Aquí lo emocional y lo emotivo significan y poseen un peso cualitativo en la comprensión de los hechos narrados. Leer y valorar el discurso de nuestro informante, provisto de la herencia emocional, hará que nos alejemos del convencional texto científico positivista, ya que está circunscrito a la prudencia interpretativa. Tal como suscribe Landín Miranda y Sánchez Trejo (2019):

el ejercicio narrativo nos permite: generar estados de reflexión y de conciencia sobre las experiencias vividas, generar una práctica para el establecimiento del diálogo que nos lleva a la develación de subjetividades en conjunto e identificar aquellos genuinos procesos educativos desde donde se ha aprendido y construido el conocimiento. (p. 229)

\section{Ética, instrumento y sujeto informante}

Y en este hecho complejo de presentar la metodología, también hemos de dar cabida a la persona informante, pues él es el sujeto activo y hacedor del proceso. Compartir la palabra y dotarlo de significado lo erige en el referente de nuestra investigación. Escucharle, activamente, nos otorga la responsabilidad de dar cabida a todos los matices de sus conversaciones (en cuatro ocasiones, en sesiones de una hora cada una). Y, en este sentido, suscribimos obrar éticamente (Roth y von Unger, 2018), siendo rigurosos y fiel a su voz. Una investigación inspirada en la confianza y respeto, así como en la no necesaria pretensión extractivista y, para ello, hubo devolución de las entrevistas para matizar lo comentado por el entrevistado. Además de contarse con un posicionamiento de confidencialidad e imparcialidad en lo investigado.

Sin olvidar el protagonismo que la entrevista tiene en esta metodología, pues es la herramienta que abre la caja de Pandora. Lo que las profesoras Phillion y He (2008) señalan como el punto de partida. Estamos ante la metodología narrativa como un proceso no concluyente, sino que se abre a las demás personas al ofrecer la posibilidad de saber más. Bolívar et al. (1998) plantean:

La narrativa expresa la dimensión emotiva de la experiencia, la complejidad, relaciones y singularidad de cada acción: frente a las deficiencias de un modo atomista y formalista de descomponer las acciones en un conjunto de variables discretas. (p. 12) 
http://doi.org/10.15359/ree.25-3.29

http://www.una.ac.cr/educare

educare@una.ac.cr

Con todo, el sujeto informante es un profesional de la educación. Tras varios intentos para establecer un día para comenzar las entrevistas, esto fue posible y empezó un proceso de explicación de lo que pretendíamos realizar y de conversaciones cuasi informales. En este sentido, cabría añadir que la primera entrevista no se grabó pues el debate fue abierto. Además, se inspiraron en la empatía y en el reconocimiento de lo que nos queda por aprender. Con todo, su sinceridad hizo que revelara que el libro La ciencia de enseñar ciencia. Desarrollo cognoscitivo y exigencias del currículo, de Shayer y Adey (1984), le ayudó en sus pasados años como profesor novel.

Las tres entrevistas semiestructuradas, que prosiguieron, sí fueron grabas y, sin género de dudas, fue un momento de intercambio y de enriquecimiento en el que se asistió a experimentar cómo la emotividad se apoderaba del discurso. Era la consecuencia del entusiasmo propio de la conversación y de los temas tratados. Las entrevistas se introdujeron en la dinámica de la investigación, según Denzin y Lincoln (2005), como una conversación que es el arte de realizar preguntas y escuchar respuestas.

Con todo, lo que se pretende es explicar de otra manera, si cabe, unos principios de la ciencia, a través de la didáctica, la pedagogía, la historia o la filosofía de la ciencia y, también, con la narrativa. En palabras de Haraway (2004, p. 33), una mirada con un resultado de difracción con la intención de desplazar "lo mismo ... en otro lugar"; con un resultado multidireccional. Estamos ante la inflexión intencionada que produce una "desviación" con una intención interpretativa (Denzin y Lincoln, 2003).

\section{Fases y procedimiento}

Tal vez, en las ciencias sociales no todo se pueda explicar a través de las evidencias empíricas, disponibles y objetivas, que llevan a demostrar determinada teoría o hipótesis. También se ha de dar a comprender el contexto cultural y social, además de cómo se vivió o sintió determinado hecho o saber científico. Máxime cuando nos estamos desenvolviendo en las ciencias sociales, ante una profunda complejidad, a tenor de la influencia de los hechos culturales y los, propiamente, sociales. La información cualitativa inspirada en el diálogo, el contar con las vivencias personales, la relación etnográfica... explican y dan a comprender de otra manera. En palabras de Coffey y Atkinson (2003), estamos ante una metodología del diálogo.

Si la metodología narrativa tiene como precepto el acto compartido, este se inició desde los primeros momentos en que surge la idea de entrevistarle y continuar con la revisión y quehacer explicativo. La organización y reorganización se apoderó del proceso y las conclusiones se obtienen a partir de consideraciones donde la temática se reflexiona con atención y se comparte con delicadeza con la intención, insistimos en ello, de conocer y comprender.

Las fases que mantiene la presente investigación están inspiradas en las formuladas por Rodríguez (1999), quien atisba cuatro momentos: A) preparatoria, de lecturas previas y prediseño 
http://doi.org/10.15359/ree.25-3.29

de la investigación, incluyéndose la selección del informante, elaboración de entrevista y su validación por sujetos expertos. B) trabajo de campo, con las entrevistas a la persona informante. C) analítica, a partir de los datos obtenidos, con su organización y comienzo del proceso de análisis de la información. D) informativa, donde se comprende y se da a comprender, a tenor de la obtención de los resultados, la verificación de las conclusiones y redacción del trabajo.

Por último, indicar que, siguiendo a Cornejo et al. (2017), el procedimiento metodológico se centrará a partir de determinados agrupamientos temáticos, que funcionan a modo de núcleos, derivados de la propia entrevista: didáctica de la ciencia, cultura y conocimiento científico, así como la educación de la ciencia, entre otros. En este sentido, Misischia (2020) lo plantea de la siguiente manera:

Así se aleja de categorías conceptuales, de la pretensión de describir y se acerca a la conformación de significados. No buscan la sistematización de experiencias, sino recuperar los sentidos que en ellas emergen; no se concibe un esquema previo a partir del cual organizar el conocimiento, sino que se construye en el diálogo entre la posición subjetiva del investigador con lo investigado. (pp. 72)

\section{Resultados}

\section{Núcleo temático I. Del saber y la didáctica de la ciencia}

Partimos del principio establecido por el propio informante, quien sostiene que: para saber no es necesario la importación del método científico para que este tuviera una validez académica; pues, a veces, ni la propia ciencia funciona con verdades demostrables, sino con la organización de nuevos datos. Y lo continuamos a partir del hecho tripartido de escuchar (infinitivo que hacemos coincidir con la acción de atender con atención a lo que se oye, con el propósito de alentar un aviso), observar (verbo vinculado al examen enjuiciado, con la pretensión de reparar a lo que estamos atendiendo) y comprender (o sea, la labor de discernir, pero con la convicción de encontrar justificados los hechos narrados y los sentimientos expresados). Tres quehaceres que serán nuestros referentes a la hora de llevar a cabo la pertinente acción de atender a los resultados. Es decir, estamos ante un ejercicio práctico que nos ayudará a validar el proceso de investigación narrativo (Flick, 2007).

En este marco de escucha, atención y comprensión, nuestro informante nos introduce en el debate a partir de si en la formación del profesorado, sea en nuestras facultades o en el máster de educación secundaria que se oferta en cada Universidad, lo que debemos es dar lo último en didáctica de la ciencia, o si ello no es suficiente, para que el alumnado adquiera una formación integral. Con todo, la formación integral en ciencia será un tópico recurrente en la educación, más allá del conocimiento de la ciencia o su aplicación en la vida. 
http://doi.org/10.15359/ree.25-3.29

http://www.una.ac.cr/educare

educare@una.ac.cr

Y él sostiene que: La didáctica de la ciencia no es algo terminal, algo acabado, hemos de reivindicar que el alumnado participe en el proceso y de la cultura científica, alejado de la instrucción o el adoctrinamiento. Es más, hace falta una alfabetización en ciencia, ya que la analogía que presenta nuestro informante se centra en que aquellas personas que, por ejemplo, en el siglo pasado, no hubiesen leído El Quijote eran consideradas analfabetas. Y, hoy en día, el que no tenga conocimientos científicos puede llegar a ser admitido como una persona inculta en el siglo XXI, pues, tal como sostiene él mismo, su incapacidad está en no poder emitir una opinión fundamentada de la ciencia. Y a partir de este momento el entrevistado establece una dicotomía entre ciencia y humanidades:

Ambas son parte de la cultura y de la formación ciudadana y, por ello, me gusta hablar de cultura científica con la posibilidad de trasladarlo a la vida diaria; por ejemplo, siendo capaz de leer e interpretar en un periódico una noticia de carácter científico o relacionar un programa de televisión o una información en Internet con lo que pasa en la vida cotidiana. Pues, en el fondo, ha de saber interpretar ese conocimiento científico para comprender el mundo.

De manera coherente, el entrevistado une ciencia con tecnología, al igual que cultura y formación ciudadana. Pero siempre con la tónica de saber interpretar en pleno siglo XXI el conocimiento científico; un modo de comprender y forma parte del mundo activamente. Y ahí la didáctica adquiere su protagonismo.

\section{Núcleo temático II. Del conocimiento y la educación científica}

Y, todo ello, lleva a la reconsideración de la enseñanza de la ciencia (experimental) y atenderla como un conocimiento diferenciado, pero, no por ello, superior. En este sentido, el alumnado ha de valorar este conocimiento y tener presente las posibles limitaciones que se puedan ocasionar, pues el conocimiento científico no da respuesta a cualquier problema y, por ello, no ha de llegar a verse la ciencia como una nueva religión. Igualmente, el informante hace hincapié en la idea de que se vea el conocimiento científico como algo dinámico y no estático, que evoluciona y que, en muchas ocasiones, no ofrece una verdad absoluta, sino que son aproximaciones $y$, por ello, es susceptible de mejorar o de cambiar. Con todo, la ciencia no es una doctrina por aprender; sino que se trata de un proceso.

El informante se centra en la necesaria formación para la ciudadanía en ciencia y, de este modo, crecer como sujeto activo en una sociedad democrática y procomunitaria. Sin embargo, es consciente de las fricciones de intereses con respecto a la ciencia, aspecto que explicaría la casi inexistencia del cambio e innovación en materia de educación científica y que hace evidente el desfase entre la educación para la vida y las exigencias del currículo (Hayes Jacobs, 2014).

Sin embargo, no se elude la necesidad de una educación científica para la ciudadanía que vaya en consonancia con el fomento de las ciencias y la formación para que haya buen personal científico en el futuro, incentivando en las escuelas e institutos, la disposición y el aprendizaje de 
http://doi.org/10.15359/ree.25-3.29

las ciencias. Con todo, se está impulsando el desarrollo de los valores científicos presentándolos como un producto humano, atractivo y seductor, desprotegiéndolos de aquellos estereotipos elitistas que poco, o nada, le aportan.

Seguidamente, el informante reivindica, de nuevo, como vehículo articulador en esto de la educación científica. Y apunta que la necesidad de que la didáctica de la ciencia deje de ser una gran desconocida, para llegar a convertirse en un espacio de yuxtaposición entre la didáctica y las ciencias. En este sentido, no hay que verla como la suma del conocimiento didáctico-pedagógico y el científico-experimental, sino atenderla como una disciplina propia, fruto de la complejidad y del exclusivo estatus del conocimiento didáctico. Por ello, se ha de hacer notar el papel de la didáctica de la ciencia, según este profesor, como:

Un eje articulador y fundamental entre la formación psico-pedagógica-didáctica y la formación en ciencia, y digo lo de eje no como una parte poco importante, pues el eje puede ser pequeño, pero sin su existencia aparecerían las dos partes por separado. Además de atenderla como un instrumento para focalizar los problemas docentes desde el punto de vista de los contenidos, las competencias, sus visiones previas o cómo piensan sobre los fenómenos naturales. Es decir, su comprensión en cuanto a sus planteamientos personales y alternativos.

La educación científica es imprescindible, no solo por ser motivo de un tema actual y atractivo sino, también, por la dimensión formativa que ofrece, en el amplio sentido del término. Una manera de manifestarse la lucidez que ayuda a superar la verdad absoluta y contribuye a la comprensión y valoración de la ciencia y su conocimiento. Con todo, se hace imprescindible saber interpretar el conocimiento científico para comprender el mundo y no quedar o convertirnos, irreversiblemente, en personas neoexcluidas del siglo XXI. Y, de nuevo, la didáctica de las ciencias tiene su papel.

\section{Bloque temático III. Del mensaje y del saber de la ciencia}

El entrevistado continúa diciendo: el mensaje científico puede plantear una complejidad que entra en conflicto con aquello que, a priori, se pensaba de determinado hecho o fenómeno científico. Con todo, prosiguiendo con el parecer del informante, en esta ocasión sobre el aprendizaje de la ciencia, apunta a que:

No es solo la adquisición o saber sobre unos hechos o tener más información sobre éstos, sino que se trata de estructurarlos de una manera diferente pues, en muchos casos, el alumnado ya viene con información del mundo, de los diferentes fenómenos de la vida diaria e, incluso, viene con una interpretación de esos fenómenos o con una mini teoría alternativa del mundo, lo que se llama las ideas o concepciones explicativas de los estudiantes, que son modelos intuitivos que ellos tienen de cómo funciona la naturaleza. Y ahora lo que se trata no es en persistir en la transmisión de más información sino, insisto, en que esa información la interpreten desde otro punto de vista distinto, desde otro modelo. 
http://doi.org/10.15359/ree.25-3.29

http://www.una.ac.cr/educare

educare@una.ac.cr

Y termina sosteniendo, con el matiz centrado en la revolución científica, que se trata de una necesidad de evolución de la propia humanidad, que:

Es algo parecido a lo que sucede, en ocasiones, en la historia de la ciencia, donde el científico tiene una explicación del mundo y se produce, en un momento dado, una revolución científica. Es decir, que a través de una serie de razones de ámbitos culturales o sociales que, a veces, no son ni tan siquiera empíricas, se empieza a explicar dentro de la ciencia el mismo fenómeno desde otro punto de vista y ello da lugar a esa revolución científica.

Una perspectiva que nutre el discurso del entrevistado. Siempre con la condición de dejarlo abierto para que el lector los termine de formular y resolver. Y, además, en el caso del alumnado que proviene con apriorismo que, puede o debe, cambiar en el seno de la dinámica de aula, a partir de la experiencia o la lectura, la reflexión o los puntos de vistas del docente y de sus compañeros. Un saber de la ciencia que se ha de presentar de manera integral y que no establezca separación entre ciencia y humanidades, ya que la propia ciencia es una construcción humana y no un producto; a todas luces, un valor en alza.

\section{Bloque temático IV. De cultura y alfabetización científica}

Estamos ante una cultura científica cercana a los valores y que se aleja de lo, meramente, instrumental o de la instrucción. Según nuestro profesor, hemos de comenzar a hablar con propiedad de la cultura científica:

Viene a introducir al alumnado en el conocimiento científico pues pensamos en estudios posteriores, ya que se sostiene en un enfoque propedéutico; sin embargo, no es lo más importante. Realmente lo importante es la educación científica que posee una connotación más amplia que abarcaría la formativa en ciencia o de estudio en ciencia, pero, también, aquella educación que le facilite la participación en una sociedad democrática y responsable.

Para ello, sostiene la importancia de acceder a una alfabetización científica de la misma manera que hace falta tener alfabetización en lecto-escritura. Hoy en día, en la sociedad actual, cualquier persona debe tener conocimientos sobre la ciencia para, según su opinión, poder interaccionar con el mundo y poder entender lo que sucede y, de este modo, empezar a participar como ciudadana. Es decir, de la cultura científica a la educación científica, pasando por la alfabetización de la esta misma. Pero una alfabetización, en sus propias palabras, para que:

El ciudadano participe de en una sociedad democrática, por ejemplo, conociendo aspectos básicos concernientes a la clonación, a las células madre, al aborto, a la energía nuclear o a la energía renovable, etc. Siempre para que pueda opinar y participar e, incluso, en las elecciones políticas para que no sea un voto condicionado sino con convicción propia. Y ahí radica, también, la formación y cultura científica. 
En este último bloque se suscribe el valor que posee el conocimiento científico, así como la necesidad de que el sujeto esté alfabetizado en esta materia. Con todo, se ha de superar un estudiantado pasivo que queda relegado a lo meramente anecdótico. Es decir, precisa estar actualizado conforme parte de la transición de la cultura científica a la educación científica, pasando por la alfabetización. Y, por ello, se plantea la necesidad de alfabetizar y educar, científicamente, no solo al alumnado sino, también, a la ciudadanía de forma proactiva y participativa en conexión con la vida actual, para que formen parte de la cultura científica de forma acertada y diligente.

A renglón seguido, después de esbozar los pareceres del sujeto informante, estamos en disposición de comenzar el último de los epígrafes del presente artículo, en el cual se dará pie al marco de las conclusiones. Ciertamente, un apartado en continua construcción y, por ello, la invitación se centra en el hecho de considerar; pues es una forma de seguir reflexionando.

\section{Conclusión}

La narrativa nos aporta dos reclamos de aproximación a una realidad: es decir, sobre la vida de quien narra su sentir de modo individual e, igualmente, muestra un reflejo de una vivencia colectiva, con los demás, que han configurado parte de su realidad. Esta es compartida con el investigador de este estudio, quien, a su vez, lo da a comprender. No obstante, e insistimos en ello, este último punto es un ejercicio para dar a pensar y razonar en unión con el público lector. Sostenemos una interacción con la información que nos transmite el sujeto informante, su contexto de intervención, sus pareceres $y$, además, quedamos persuadidos con el pensamiento de que los textos de ciencias sociales necesitaban construir una relación diferente entre quienes investigan y los sujetos de investigación, entre quienes escriben y quienes leen (Ellis y Bochner, 2000).

La ciencia no está muerta ni en manos de quienes la protagonizan o la escriben. Ella nos pertenece y hemos de comprenderla no como una verdad absoluta, sino, y en este contexto que nos desenvolvemos, como el resultado de un conocimiento que incentiva el pensamiento y la acción pro social y pro educativa. Nuestro quehacer, vinculado a la consideración, es un ejercicio donde contribuimos a la opinión, pero no damos por sentenciado un proceso a través de un informe. La obra en construcción es algo que el público lector cerrará o no, según su capacidad o interés. Nosotros nos centramos en un horizonte de indagación y comprensión. Por ello, escuchar la historia de otro es a menudo la manera más sobrada y profunda de reconocer los vínculos que compartimos como seres humanos (Ettling, 1998).

La acción de considerar posee varias acepciones. Cabría hablar de un verbo transitivo con el sentido de juzgar o estimar. Pero nuestra propuesta se abre hacia un espacio para reflexionar y analizar, pensar y discurrir, razonar e, incluso, imaginar. Por ello, incentivamos una formación en la cultura científica para crecer como persona, en el ámbito de su desarrollo integral que, 
http://doi.org/10.15359/ree.25-3.29

http://www.una.ac.cr/educare

educare@una.ac.cr

según nuestro informante, está vinculado con la ciudanía, la participación, la solidaridad y la democracia, alejado del individualismo y acercándonos al espíritu colaborativo. E, igualmente, el entrevistado lo hace coincidir con la educación en valores y suscribe el desarrollo integral de la persona para que tengan una visión certera e idónea del mundo en que vivimos; aunque, en este momento, prevalece más el individualismo que lo colectivo. Aunque el informante solicita para paliar el envite del consumo, una inmersión en la cultura científica, para saber hasta dónde podemos llegar o hacia dónde vamos, qué intereses hay o qué nos ocultan...

La concepción de ciencia pasa por cuatro segmentos de intervención: a) aprender ciencia, b) hacer ciencia, c) comprender la ciencia y d) utilizar la ciencia para coexistir en una sociedad democrática. Todo son partes de un discurso que persigue la presencia e implicación en el tiempo en presente, para que ese conocimiento le sirva para participar y, además, de ser una necesidad social en cuanto al conocimiento y el desarrollo del juicio crítico. Todo esto viene a cuestionar la enseñanza tradicional de la ciencia dirigida a la formación de futuro personal científico... Y se incentiva una visión más contemporánea, en relación con las demandas sociales, económicas o ambientales.

La respuesta educativa se centra en los proyectos de investigación y en la propia didáctica de la ciencia con el movimiento CTS (ciencia, tecnología y sociedad), a la que habría que incluir la A de ambiental (López Cerezo y Sánchez Ron, 2001). Y este concepto hace evolucionar la idea inicial de enseñanza por el de educación científica. No obstante, existen tres referentes pendientes en este proceso:

a) La formación del profesorado en didáctica, aunque habría que matizar que en primaria es el maestro o maestra quien tiene los conocimientos didácticos, pero, tal vez, cuenta con unas carencias en la formación científica o ambiental y, en secundaria, resulta al contrario, por lo cual se tiene que cuidar el tránsito de primaria a secundaria. Y será en la ESO donde se mantiene la preocupación de formar a personal científico vehiculizándolo hacia la universidad.

b) La visión de una ciencia más educativa que no se da en la institución y que algunos padres y madres no terminan de entender, pues insisten en la necesidad de que sus hijos e hijas reciban instrucción más que educación; a quienes mueve la inmediatez y las exigencias de un posible futuro profesional de familia.

c) La existencia de estándares de exigencia como podría ser la selectividad (pruebas de acceso a la universidad) promueve que gran cantidad de docentes enseñen pensando para este examen. Y, por extensión, hemos de hablar del currículo que se elabora con una finalidad para la instrucción. El fallo que se está manteniendo es separar la ciencia de las humanidades, por ejemplo, de la historia, sus relaciones y contribuciones para entender la ciencia dentro del contexto, pues la ciencia es una construcción humana y no un producto. Al respecto, Hernández Guerrero (2015) sostiene: 
http://doi.org/10.15359/ree.25-3.29

Aprender a vivir humanamente es una tarea complicada $y$, a veces, una asignatura pendiente que no sólo se ha de aprobar, sino que, además, se ha de colaborar para que los otros también la aprendan. ... Concibo, por lo tanto, la educación no como mera "instrucción", como unos quehaceres en los que los destinatarios son meros receptores de información (tanto de las ciencias como de las letras), sino como la "construcción" de unos modelos de comportamientos adaptados a las sucesivas necesidades surgidas durante el proceso de desarrollo del individuo y de la sociedad que no se reducen al traslado de datos sobre los fenómenos económicos, técnicos y científicos que vayan emergiendo. (pp. 35-36)

En este entramado de carencias y deseos se manifiesta la necesidad de una educación científica ciudadana, pues la ciencia está en nuestras vidas y algunas de estas explicaciones científicas, en ocasiones, no son muy complejas. Es decir, promover una propuesta de relación de lo que se aprende con la cotidianeidad y romper con la estricta formación científica, vinculada al plano académico, sin conexión con la vida; que explique, interprete o prediga lo cotidiano (y el futuro inmediato). Pero, igualmente, se hace necesario un ejercicio por divulgar la ciencia en la escuela, y fuera de esta, con el propósito de interactuar con los conocimientos de las ciencias básicas que permitan desde interpretar una etiqueta de una botella de agua, hasta disfrutar con los contenidos de una película de ciencia-ficción. Es decir, una educación científica ciudadana que, además de comprender, sirva para interactuar con el medio en que vivimos. Sin olvidarse de una educación en estos ámbitos para una ciudadanía proactiva y participativa, capaz de tomar decisiones en propuestas científicas, vinculadas con la política, lo social o lo económico... $y$, hasta, con lo ambiental.

El informante, en cierto modo, propone un cambio en la perspectiva de la didáctica de la ciencia. Por consiguiente, del saber científico, con una visión o modelo explicativo que se ha de adecuarse a la realidad. Y, por parte del alumnado, contribuir y participar del conocimiento, superándose el unicista modelo transmisivo inspirado en la función del personal docente. Entonces, aprender ciencia no es precisamente adquirir más información, sino que consistiría en una restructuración de la que se tiene $y$, con ello, verla/aprovecharla desde y con otro punto de vista. Igualmente, en este ejercicio de comprensión, la propuesta se centra en desmitificar la ciencia, quitándole ese halo de proceder lejano y distante, que se elabora tan solo en sofisticados laboratorios por personas muy cualificadas, lo cual produce una fractura entre alumnado, y ciudadanía en general, con la propia ciencia. Se sugiere, al contrario, la introducción de una ciencia como algo cotidiana que se ha de valorar y conocer pues está ahí en la vida, conformando parte de ella, explicándola... Y que el alumnado participe de esa explicación, ya que le enriquece y le permite conocer y entender mejor el mundo en el que cohabita.

En virtud del objetivo marcado y las cuestiones de investigación establecidas, la presente investigación da respuesta a planteamientos como la necesidad de desarrollar un modelo de cultura científica para crecer como persona y que sea capaz de generar un conocimiento cercano 
http://doi.org/10.15359/ree.25-3.29

http://www.una.ac.cr/educare

educare@una.ac.cr

a los valores (sociales y ambientales). Se plantea la posibilidad de alejarse de la educación instrumental o de la instrucción. E, igualmente, se aboga por una educación y saber científico que supere el modelo transmisible por otro más comprensivo y cotidiano, que permita comprender.

\section{Declaración de Material complementario}

Este artículo tiene disponible, como material complementario:

-La versión preprint del artículo en https://doi.org/10.5281/zenodo.4796705

\section{Referencias}

Acevedo Díaz, J. A. (2010). Formación del profesorado de ciencias y enseñanza de la naturaleza de la ciencia. Revista Eureka sobre Enseñanza y Divulgación de las Ciencias, 7(3), 653-660. https://revistas.uca.es/index.php/eureka/issue/view/199

Aguilera Morales, D., Martín-Páez, T., Valdivia-Rodríguez, V., Ruiz-Delgado, Á., Williams-Pinto, L., Vílchez-González, J. M. y Perales-Palacios, F. J. (2018). La enseñanza de las ciencias basada en indagación. Una revisión sistemática de la producción española. Revista de Educación, (381), 259-274. https://doi.org/10.4438/1988-592X-RE-2017-381-388

Arfuch, L. (2007). El espacio biográfico. Dilemas de la subjetividad contemporánea. Fondo de Cultura Económica.

Arteaga Valdés, E., Armada Arteaga, L. y del Sol Martínez, J. L. (2016). La enseñanza de las ciencias en el nuevo milenio. Retos y sugerencias. Universidad y Sociedad, 8(1), 169-176. https://rus. ucf.edu.cu/index.php/rus/article/view/321/318

Ballesteros Velázquez, B. (2019). Investigación social desde la práctica educativa. UNED.

Blanco, C. (2015). Lógica, ciencia y creatividad. Dykinson.

Bolívar, A., Domingo, J. y Fernández, M. (1998). La investigación biográfico-narrativa en educación. Guía para indagar en el campo. FORCE. https://www.researchgate.net/ publication/286623877 La investigacion biografico-narrativa Guia para indagar en el campo

Coffey, A. y Atkinson, P. y (2003). Encontrar el sentido a los datos cualitativos. Estrategias complementarias de investigación. Editorial Universidad de Antioquia.

Cornejo, M., Faúndez, X. y Besoain, C. (2017). El análisis de datos en enfoques biográficosnarrativos: Desde los métodos hacia una intencionalidad analítica. Forum: Qualitative Sozialforschung/Forum:Qualitative Social Research, 18(1), 1-25.http://dx.doi.org/10.17169/ fqs-18.1.2491 
Decuir-Gunby, J. T., y Schutz, P.A. (2017). Asking appropiate research questions. En J. T. DecuirGunby y P.A. Schutz (Autores), Developing a mixed methods proposal: A practical guide for beginnig reserachers (pp. 33-43). Sage.

De Pro Chereguini, C., Inglés Carrillo, C. y Gómez Mármol, A. (2020). ¿Qué aprenden unos estudiantes en una experiencia formativa de didáctica de las ciencias experimentales en el Grado de Educación Infantil? Didáctica de las Ciencias Experimentales y Sociales, (38), 97113. https://doi.org/10.7203/dces.38.16174

Denzin, N. K. y Lincoln, Y. S. (2003). The landscape of qualitative research. Theories and issues. Sage.

Denzin, N. K. y Lincoln, Y. S. (2005). The sage handbook of qualitative research (3. a ed.). Sage.

Ellis, C. y Bochner, A. P. (2000). Autoethnography, personal narrative, reflexivity: researcher as subject. En Denzin, N. K. y Lincoln Y. S. (Eds.), Handbook of qualitative research (pp. 733768). Sage.

Ettling, D. (1998). Levels of listening. En W. Braud y R. Anderson (Eds.), Transpersonal research methods for the Social Sciences. Honoring human experience (pp. 176-179). Sage.

Flick, U. (2007). Introducción a la investigación cualitativa. Morata.

Freire, P. (2005). Pedagogía del oprimido. Siglo XXI.

Galagovsky Kurman, L. (2011). Didáctica de las ciencias naturales: El caso de los modelos científicos. Lugar Editorial.

Haraway,D.J.(2004).Testigo_modesto@segundo_milenio.hombrehembra@_Conoce_Oncoratón ${ }^{\circledR}$ Feminismo y tecnociencia. Editorial UOC.

Harding, S. (2007). Feminist standpoints. En S. Hesse-Biber (Ed.), Handbook of Feminist research. Theory and praxis (pp. 45-69). Sage.

Hayes Jacobs, H. (Ed.). (2014). Currículum XXI. Lo esencial de la educación para un mundo en cambio. Narcea.

Hernández Guerrero, J. A. (2015). La pluridisciplinariedad de la enseñanza de la lectura y de la escritura. Hachetetepé. Revistga Científica de Educación y Comunicación, (11), 29-42. https:// doi.org/10.25267/Hachetetepe.2015.v2.i11.4

Landín Miranda, M. del R. y Sánchez Trejo, S. I. (2019). El método biográfico-narrativo. Una herramienta para la investigación educativa. Educación, 28(54), 227-242. https://doi. org/10.18800/educacion.201901.011

López Cerezo, J. A. y Sánchez Ron, J. M. (Eds). (2001). Ciencia, tecnología, sociedad y cultura en el cambio de siglo. Biblioteca Nueva y Organización de Estados Iberoamericanos. 
http://doi.org/10.15359/ree.25-3.29

http://www.una.ac.cr/educare

educare@una.ac.cr

Michel Sandoval, B. y García Guerrero, M. (Coords.). (2014). La ciencia en nuestras manos. Una perspectiva de los talleres de divulgación sin el color de rosa. Texere

Misischia, B. S. (2020). Formación y narrativa. Núcleos de sentido a partir del recorrido autobiográfico. Márgenes Revista de Educación de la Universidad de Málaga, 1(3), 63-77. https://doi.org/10.24310/mgnmar.v1i3.8355

Moriña, A. (2017). Investigar con historias de vida: Metodología biográfico-narrativa. Narcea.

Parker, J. (2006). Exploring the impact of varying degrees of cognitive conflict in the generation of both subject and pedagogical knowledge as primary trainee teachers Learn about shadow formation. International Journal of Science Education, 28(13), 1545-1577. https:// doi.org/10.1080/09500690600780179

Phillion, J. y He, M. F. (2008). Multicultural and cross-cultural narrative inquiry in educational research. Thresholds in Education, 34(1-2), 2-12 https://academyedstudies.files.wordpress. com/2015/09/2008completethrvol34no12.pdf

Pujolàs Maset, P. (2008). 9 ideas clave. El aprendizaje cooperativo. Grao.

Ovejero Bernal, A. (2018). Aprendizaje cooperativo crítico: Mucho más que una eficaz técnica pedagógica. Pirámide.

Roth, W.-M. y von Unger, H. (2018). Current Perspectives on Research Ethics in Qualitative Research. Forum Qualitative Sozialforschung / Forum: Qualitative Social Research, 19(3) 1-12. http://dx.doi.org/10.17169/fqs-19.3.3155

Shayer, M. y Adey, P. (1984). La ciencia de enseñar ciencias. Desarrollo cognoscitivo y exigencias del currículo. Narcea.

Simon Auerbach, J. (2019). The teaching and learning of science in secondary education. CLIL Journal of Innovation and Research in Plurilingual and Pluricultural Education, 2(2), 67-71. https://doi.org/10.5565/rev/clil.17

Vázquez-Bernal B., Jiménez-Pérez, R. y Mellado Jiménez, V. (2019). El conocimiento didáctico del contenido (CDC) de una profesora de ciencias: Reflexión y acción como facilitadores del aprendizaje. Enseñanza de las ciencias, 37(1), 25-53. https://doi.org/10.5565/rev/ ensciencias. 2550 